\title{
Does it matter what we measure? Core outcomes, the IUJ and the CROWN and COMET initiatives
}

\author{
P. L. Dwyer • P. Riss • B. T. Haylen
}

Published online: 30 July 2014

(C) The International Urogynecological Association 2014

Khalid Khan, the Editor-in-Chief of the British Journal of Obstetrics and Gynaecology, is the driving force behind the CROWN (CoRe Outcomes in WomeN's Health) Initiative to develop an agreed standardized collection of outcomes for all clinical trials. The need for this has been recognized by the editors of over 50 journals related to women's health [1], including the International Urogynecology Journal. An initial step in this process is the simultaneous publication of Dr. Khan's CROWN Initiative editorial [2] in all contributing journals.

However, standardizing core outcomes will be only one part of any possible solution. This needs to go hand in hand with standardization of terminology for symptoms, signs, assessment standardization and definitions (e.g. POPQ), investigation modalities including imaging, diagnoses and finally treatment terminology for both conservative and surgical options. How can you assess outcomes if you have not achieved agreement on the diagnosis or the assessment tools or defined and standardized treatment options?

In the area of female pelvic floor dysfunction (PFD), including urinary incontinence and pelvic organ prolapse (POP), much of this work has already been completed

\footnotetext{
P. L. Dwyer $(\bowtie)$

Department of Urogynecology, Mercy Hospital for Women, University of Melbourne, Melbourne, Austria

e-mail: pdwyer@connexus.net.au

P. Riss

Department of Obstetrics \& Gynecology, Medical University

Vienna, Vienna, Austria

e-mail: priuj11@gmail.com

B. T. Haylen

St Vincent's Clinic \& University of New South Wales, Sydney, Australia

e-mail: bernard@haylen.co
}

by the standardization committees of the International Urogynecology Association (IUGA) and the International Continence Society (ICS) [3, 4]. Documents currently in development on (i) female anorectal dysfunction and (ii) conservative management of PFD with newly initiated reports on (iii) pelvic organ prolapse and (iv) sexual health in women with PFD will supplement the four published Joint IUGA-ICS Standardization Reports [3-6].

The IUGA-ICS standardization of reporting outcomes measures for female POP surgeries has been published in this journal [4]. The aforementioned documents (ii) and (iii) will augment this work. Other IUGA work on surgical outcomes has also been published in the IUJ [7]. Outcomes need to determine the effectiveness of an intervention and usually assess both subjective and objective criteria. The safety of the intervention needs to be determined by looking at the risk of early and late complications. Complications also need classifying and standardization in a systematic process so it will possible to compare complications of different interventions. IUGA-ICS have been pro-active in such initiatives, providing classifications for surgeries involving (a) prostheses and grafts and (b) native tissues, facilitating possible comparison studies [5, 6]. As many complications have a low incidence, often very large databases are needed to determine their true incidence and significance, such as the ICS-IUGA Complications Registry (http://www.ics.org/complicationregistry) which has been developed for this purpose.

The Standardization Committees should ensure that this work continues into the future in our societies. The CROWN Initiative will highlight the importance of standardization not only of outcomes but also of these other important clinical areas to improve reporting of results and enable more accurate comparisons of trials to be made. This in time will lead to consensus not only among journals but also among clinicians, societies and the community at large. 
Every clinical study should follow the PICO process: (1) what is the problem; (2) what is the intervention; (3) what are we comparing (a control group); and (4) which outcome do we measure? The importance of the selection of the outcome cannot be overemphasized. The outcome must be of interest and it must be relevant. This is particularly true for clinical trials where we expect the results to help us in our daily clinical practice.

The selection of outcomes also depends on current thinking and how medicine is perceived. Good examples are studies of operations for pelvic organ prolapse. A landmark trial by Benson et al. published in 1996 focused on anatomical outcome and reoperation [8]. Surgical effectiveness was optimal in $29 \%$ of the vaginal group and $58 \%$ of the abdominal group; it was unsatisfactory leading to reoperation in $33 \%$ of the vaginal group and $16 \%$ of the abdominal group. Seventeen years later (2013), another surgical trial by Freeman et al. included patient-centred outcomes such as quality of life [9]. This prospective multicentre two-sided equivalence trial of open and laparoscopic sacrocolpopexy using objective and subjective outcomes showed clinical equivalence.

Researchers often choose outcomes which are readily available, easy to measure and with an expected large difference to the control group, making power calculations easier. These outcomes are not necessarily the most relevant ones and frequently vary from trial to trial, which makes it impossible to compare the results of one study to those of another. Outcomes in clinical trials must focus on the needs, the expectations and the problems of the patients, and they must be relevant for clinical practice. As a consequence all surgical trials today must include parameters reflecting the quality of life of the patient, including daily activities, urogenital symptoms and sexual function. Surgical trials must have an adequate follow-up time of at least 12 months; the longer the better. However, one trial is almost never enough to answer a question. The aggregated knowledge of several trials will be the foundation for evidence-based medicine. The tools to do this are systematic reviews and meta-analyses. Comparing different trials or aggregating the results from various trials is only possible when the same or least very similar outcome parameters are used.

The International Urogynecology Journal supports the COMET (Core Outcome Measures in Effectiveness Trials) [10] and CROWN (Core Outcomes in Women's Health) [1] initiatives. The aim is to make it easier for researchers to choose the appropriate outcomes. When their work is submitted to a peer-reviewed journal authors should not have to be afraid that the outcome parameters chosen in their study will not be recognized by reviewers. In the long run the use of core outcome parameters should facilitate systematic reviews and meta-analyses and thus raise the quality of evidence, which will be to the benefit of all of our patients.

\section{References}

1. Core Outcomes in Women's Health (CROWN) initiative. http://www.bjog.org/view/0/crown-initiative.html Accessed 10 May 2014

2. Khan K (2014) The CROWN Initiative: journal editors invite researchers to develop core outcomes in women's health. Int Urogynecol J. doi:10.1007/s00192-014-2447-1

3. Haylen BT, Freeman RM, de Ridder D, Swift SE, Berghmans B, Lee J, Monga A, Petri E, Rizk D, Sand P, Schaer G (2010) An International Urogynecological Association (IUGA) - International Continence Society (ICS) Joint Report into the Terminology for Female Pelvic Floor Dysfunction. Neurourol Urodyn 29:4-20, International Urogynecology J, 21:5-26

4. Toozs-Hobson P, Freeman R, Barber M, Maher C, Haylen B, Athanasiou S, Swift S, Whitmore K, Ghoniem G, de Ridder D (2012) An International Urogynecological Association (IUGA)/ International Continence Society (ICS) joint report on the terminology for reporting outcomes of surgical procedures for pelvic organ prolapse. Int Urogynecol J 23(5):527-535

5. Haylen BT, Freeman RM, Swift SE, Cosson M, Davila GW, Deprest J, Dwyer PL, Fatton B, Kocjancic E, Lee J, Maher C, Rizk DE, Petri E, Sand PK, Schaer GN, Webb R (2011) An International Urogynecological Association (IUGA)/International Continence Society (ICS) Joint Terminology and Classification of complications related directly to the insertion of prostheses (meshes, implants, tapes) and grafts in female pelvic floor surgery. Int Urogynecol J 22:3-15

6. Haylen BT, Freeman RM, Lee J, Swift SE, Cosson M, Deprest J, Dwyer PL, Fatton B, Kocjancic E, Maher C, Petri E, Rizk DE, Schaer GN, Webb R (2012) An International Urogynecological Association (IUGA)/International Continence Society (ICS) joint terminology and classification of the complications related to native tissue female pelvic floor surgery. Int Urogynecol 23:515-526, /Neurourol Urodyn 31:406-414. (dual publication)

7. Ghoniem G, Stanford E, Kenton K, Achtari C, Goldberg R, Mascarenhas T, Parekh M, Tamussino K, Tosson S, Lose G, Petri E (2008) Evaluation and outcome measures in the treatment of female urinary stress incontinence: International Urogynecological Association (IUGA) guidelines for research and clinical practice. Int Urogynecol J Pelvic Floor Dysfunct 19:5-33

8. Benson JT, Vincent Lucente V, McClellan E (1996) Vaginal versus abdominal reconstructive surgery for the treatment of pelvic support defects: A prospective randomized study with long-term outcome evaluation. Am J Obstet Gynecol 175:1418-1422

9. Freeman RM, Pantazis K, Thomson A, Frappell J, Bombieri L, Moran P, Slack M, Scott P, Waterfield M (2013) A randomised controlled trial of abdominal versus laparoscopic sacrocolpopexy for the treatment of post-hysterectomy vaginal vault prolapse: LAS study. Int Urogynecol J 3:377-384

10. COMET - Core Outcome Measures in Effectiveness Trials initiative. http://www.comet-initiative.org/ Accessed 10 May 2014 